Check for updates

Cite this: RSC Adv., 2019, 9, 31621

Received 15th August 2019

Accepted 17th September 2019

DOI: $10.1039 / c 9 r a 06366 h$

rsc.li/rsc-advances

\section{Theoretical study on the excited state decay properties of iron(II) polypyridine complexes substituted by bromine and chlorine $\uparrow$}

\begin{abstract}
Yuan Li, Xue-Wen Fan, Jie Chen, Fu-Quan Bai (D)* and Hong-Xing Zhang*
Transition metal iron(॥) polypyridyl complexes with quintet ground states were deeply investigated by density functional theory (DFT) and time-dependent density functional theory (TDDFT). Compared with the parent complex $\left[\mathrm{Fe}(\mathrm{tpy})_{2}\right]^{2+}$ (tpy $=2,2^{\prime}: 6^{\prime}, 2^{\prime \prime}$-terpyridine), the ground states of the complexes substituted by halogen atoms changed from singlet states to quintet states with rare high spin excited state lifetimes. The substituted complex $\left[\mathrm{Fe}(\mathrm{dbtpy})_{2}\right]^{2+}$ (1) results in a high spin metal-ligand charge transfer lifetime of $17.4 \mathrm{ps}$, which is $1.4 \mathrm{ps}$ longer than that of $\left[\mathrm{Fe}(\mathrm{dctpy})_{2}\right]^{2+}(2)$ with the substitution of chlorine atoms. The reason for this is explored by a combination of electronic structures, absorption spectra, extended transition state coupled with natural orbitals for chemical valence (ETS-NOCV) studies and potential energy curves (PECs). The distortion of 1 in the angles and dihedrals of the ligands is slightly larger than that in 2, although the average metal-ligand bond lengths of the latter are larger. The twisted octahedron decreases the interactions between the $d$ orbitals of iron(॥) and the $n / \pi$ orbitals of the ligands. Compared with 2, the enlarged energy gaps among the different PECs of 1 and the increased energy crossing points caused by the larger distortion result in the increase of its excited state lifetime. The different pairwise orbital interaction contributions between the metal center and the ligands in their singlet states are qualitatively estimated by ETS-NOCV. The results show that the substitution of bromine atoms will decrease the electrostatic attraction between the metal and ligands but not significantly impact the orbital interactions.
\end{abstract}

\section{Introduction}

Transition metal complexes have demonstrated their capabilities in organic light emitting devices (OLEDs) on account of their phosphorescence mechanism. ${ }^{\mathbf{1 , 2}}$ Their luminescence quantum efficiencies are as high as $100 \%$, and they have seen large-scale commercial production since the first phosphorescent OLED was reported in $1996 .{ }^{3}$ Most studies have been performed on precious transition metals, such as ruthenium, iridium and platinum. ${ }^{4-7}$ However, their high cost and environment pollution limit their applications. Recently, the use of inexpensive metals such as copper, zinc and iron has seen an increasing amount of studies to promote light-harvesting applications. ${ }^{8-16}$ Although iron is the second most Earthabundant metal on Earth and is inexpensive and environmentally benign, its subpicosecond nonradiative relaxation from the metal-to-ligand charge transfer (MLCT) state into a low-lying

International Joint Research Laboratory of Nano-Micro Architecture Chemistry, Laboratory of Theoretical and Computational Chemistry, Institute of Theoretical Chemistry, Jilin University, Changchun 130023, People's Republic of China. E-mail: baifq@jlu.edu.cn; zhanghx@jlu.edu.cn

$\dagger$ Electronic supplementary information (ESI) available. See DOI: 10.1039/c9ra06366h metal-centered (MC) state results in more difficult utilization. ${ }^{17,18}$ Investigations of the excited state decay path of the prototype $\left[\mathrm{Fe}(\mathrm{bpy})_{3}\right]^{2+}$ (bpy $=$ 2,2-bipyridine) complex have proved that ultrafast intersystem crossing (ISC) occurs from the ${ }^{1,3} \mathrm{MLCT}$ state to the ${ }^{3} \mathrm{MC}$ state and then to the ${ }^{5} \mathrm{MC}$ state. Increasing the ligand field strength is expected to change the energetic accessibility of the MC states, which results in a decrease in the nonradiative decay. ${ }^{19}$ Haacke and Gros et al. used N-heterocyclic carbine ligands to dramatically increase the Fe(II) MLCT excited state lifetime to 26 ps by strengthening the metal-ligand bonds. ${ }^{20}$ More recently, using a combination of cyano ligands and bpy ligands has been demonstrated as a way of separately controlling the ${ }^{3,5} \mathrm{MC}$ and ${ }^{1,3} \mathrm{MLCT}$ state energies to achieve a 20 ps lifetime. ${ }^{9}$ However, an opposite strategy of decreasing the ligand-field strength is still used to expand the charge-transfer excited state lifetimes, which increases the excited state lifetimes effectively. Damrauer et al. introduced this strategy for extending MLCT lifetimes by using different halogen atoms connected to the pyridyls which interact with the opposing ligand. ${ }^{6}$ The insertion of halogen atoms destabilizes the singlet ground state and results in a quintet ground state and an optically accessible ${ }^{5,7}$ MLCT. In this work, the conclusion is based on two ideas. Firstly, the ${ }^{5,7}$ MLCT happens to be less coupled to intermediates such as ${ }^{3} \mathrm{MC}$, which is necessary 
for nonradiative decay processes. Secondly, the sizes of the different halogen substituents diminish the conformational freedom of the system within the excited state manifold, thus preventing the dynamics necessary for intersystem crossing within the relaxation process.

It is an enormous challenge to explore the luminescence of $\mathrm{Fe}(\mathrm{II})$ complexes in detail because of their mixed multiple spin states, such as singlet, triplet, quintet and septet states. Moreover, it is difficult to detect the corresponding photophysical and decay mechanisms by experimental methods. With improving computational power and theoretical approaches, quantum chemical calculations can provide significant insights not only into the fundamental structures, but also into the electronic and photophysical properties of metal complexes. Here, we study the complexes $\left[\mathrm{Fe}(\mathrm{dbtpy})_{2}\right]^{2+}$ (dbtpy is the abbreviation of $6,6^{\prime \prime}$-dibromo-2,2'; $6^{\prime} 2^{\prime \prime}$-terpyridine), simplified to $\mathbf{1}$, and $\left[\mathrm{Fe}(\text { detpy })_{2}\right]^{2+}$ (dctpy is the abbreviation of $6,6^{\prime \prime}$ dichloro-2,2': $6^{\prime} 2^{\prime \prime}$-terpyridine), named 2 , to explore how halogen atoms increase the high spin MLCT lifetimes with larger sizes (Fig. 1). In Fig. 1, $\mathrm{N}_{\mathrm{c}}$ and $\mathrm{N}_{\mathrm{t}}$ are the central and terminal nitrogen atoms of tpy, respectively. $\mathrm{X}$ represents the halogen atoms.

When the parent complex $\left[\mathrm{Fe}(\mathrm{tpy})_{2}\right]^{2+}$ is substituted by halogen atoms, quintet ground states are accessible with rare high spin MLCT state lifetimes as long as 14-17.4 ps. The singlet ground state is favoured by enthalpy and the quintet ground state is populated due to entropic contributions to the free energy ( 3 : 97 at room temperature); thus, the fluorine-substituted complex is complicated to discuss. The special characteristic and ligand field of the F-substituted complex $\left[\mathrm{Fe}(\mathrm{dftpy})_{2}\right]$ may result from the small bulk of $\mathrm{F}$ with weaker steric hindrance of the interactions compared to $\mathrm{Cl}$ or $\mathrm{Br}$. Weakly sterically hindered interactions result in higher conformational freedom, which gives rise to an enthalpically favoured singlet ground state. Before analyzing the effects of halogen substitution in the coexistence of mixed spin states, we may obtain insights into the mechanism of the particularity of the spin crossover equilibrium after solving the decay mechanisms of $\mathbf{1}$ and $\mathbf{2}$ with pure quintet ground states well in advance in this work.

The distortion in the angles and dihedrals of the ligands of $\mathbf{1}$, increased by bromine atoms with greatly sterically hindered interaction, is slightly larger than in 2 , although the average

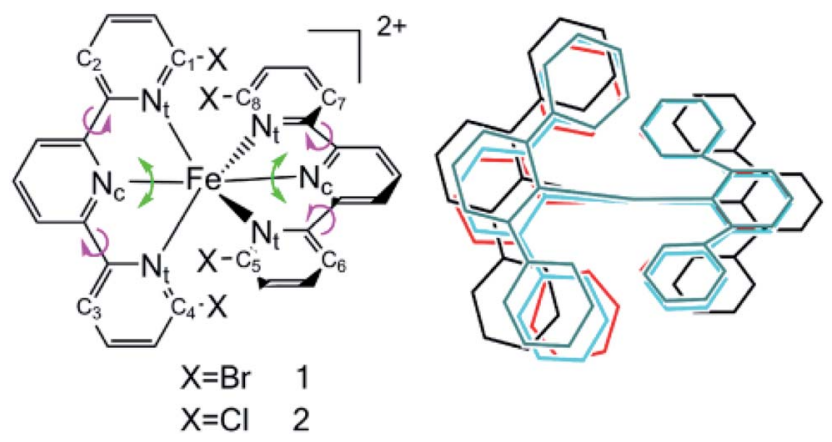

Fig. 1 (Left): Two constructions containing halogen substituents (Right): The skeletons of the fully optimized structures of 1 and 2 with halogens omitted in the ground state ${ }^{5} \mathrm{MC}$ (black), singlet state (red), triplet state ${ }^{3} \mathrm{MC}$ (cyan) and septet state ${ }^{7} \mathrm{MLCT}$ (green). metal-ligand bond lengths of the latter are larger. The increase of the CT proportion from the chlorine to bromine substituents causes hypsochromic shifts of the lowest absorption peaks. The hole and electron analysis simplifies the manifold orbitals as one main transition mode. The potential energy curves (PECs) consist of the average values of the $\mathrm{Fe}-\mathrm{N}_{\mathrm{c}}$ bond lengths as the $x$ axis and the single-point energies as the $y$-axis. This shows that the sum of the energy gaps of intersystem crossing according to the Franck-Condon rules and Kasha's rule on the PECs of the different spin states of $\mathbf{1}$ is larger than that of 2 , which results in the 1.4 ps longer lifetime. The higher energy crossing points of 1 improve the energy barriers and hinder the thermodynamic hopping from the optimized structures to the energy crossing points, which results in faster non-radiative decay. ETS-NOCV studies and the pictures of the deformation density show that the substitution of bromine atoms will decrease the electrostatic attraction between the metal and ligands but only slightly impact the orbital interactions.

\section{Computational details}

The geometrical optimizations of the ground quintet states, singlet states, triplet states and septet states of $\mathbf{1}$ and $\mathbf{2}$ were separately simulated by DFT and UDFT with the hybrid functional PBE0. ${ }^{21}$ The quasi-relativistic effective core potential (ECP) of Fe proposed by Hay and Wadt with 18 valence electrons with the double- $\zeta$ quality basis set LANL2DZ ${ }^{22}$ and the 6$31 \mathrm{G}(\mathrm{d})^{23}$ basis set for $\mathrm{H}, \mathrm{C}, \mathrm{N}$, and $\mathrm{Cl}$ and LANL08 (ref. 24) for $\mathrm{Br}$ were adopted. Vibrational frequencies were calculated at the same level to confirm that all the optimized structures were at the minimal points of their potential energy surfaces without imaginary frequencies. Furthermore, the solvent effect of acetonitrile $\left(\mathrm{CH}_{3} \mathrm{CN}\right)$ was considered by the polarized continuum model (PCM) ${ }^{25}$ for all the calculations. The absorption spectra and potential energy surfaces were simulated by LANL2TZ(f) ${ }^{26}$ and 6-311g(d, p ${ }^{27}$ for higher accuracy. The above calculations were all performed using Gaussian 16 Rev. A.03. ${ }^{28}$ The hole and electron analysis function of Multiwfn 3.6 (ref. 29) was used to analyse the complicated transition orbitals of the absorption processes.

The bond properties among the metal center, tpy and halogen atoms at different spin states were determined by the ETS-NOCV ${ }^{30-33}$ algorithm of the Amsterdam Density Functional (ADF) 2016.104 program. $^{34,35}$ The BP86 (ref. 36-38) functional with a Slater type all-electron triple- $\zeta$ basis set with polarizations $(\mathrm{TZP})^{39}$ was used with one-component zeroth-order regular approximation (ZORA) ${ }^{\mathbf{4 0 - 4 2}}$ on the scalar relativistic singlet geometries. Moreover, the conductor-like screening model (COSMO) ${ }^{43}$ of solvent with $\mathrm{CH}_{3} \mathrm{CN}$ was adopted in the ETSNOCV calculations.

\section{Results and discussion}

\subsection{Geometrical structures}

The main optimized geometrical parameters of the different spin excited states of $\left[\mathrm{Fe}(\mathrm{tpy})_{2}\right]^{2+},{ }^{\mathbf{4 4}}\left[\mathrm{Fe}(\mathrm{dbtpy})_{2}\right]^{2+}(\mathbf{1})$ and $\left[\mathrm{Fe}(\mathrm{dbtpy})_{2}\right]^{2+}$ (2) are listed in Table 1 with the corresponding experimental X- 
ray characterization results. The calculated data of $\mathrm{Fe}-\mathrm{N}_{\mathrm{c}}, \mathrm{Fe}-\mathrm{N}_{\mathrm{t}}$ and $\mathrm{N}_{\mathrm{t}}-\mathrm{N}_{\mathrm{c}}-\mathrm{N}_{\mathrm{t}}$ are in good agreement with the experimental values, with deviations of $0.9 \%$ to $2.4 \%$. Also, the quintet metalcenter states of $\mathbf{1}$ and $\mathbf{2}$ have the lowest energies compared with the singlet, triplet and septet states.

In the ground quintet states, the bond lengths between the nitrogen atoms and iron(II) center are longer than those of the other spin excited states. The bond lengths of $\mathrm{Fe}-\mathrm{N}_{\mathrm{c}}$ of the quintet states, triplet states and singlet states of $\mathbf{1}$ and $\mathbf{2}$ are separately $2.11 / 2.13,1.93$ and $1.90 \AA$. Meanwhile, the bond lengths of $\mathrm{Fe}-\mathrm{N}_{\mathrm{t}}$ have the same trend as those of $\mathrm{Fe}-\mathrm{N}_{\mathrm{c}}$. When they have the same spin states, there are only 0 to $0.02 \AA$ deviations between Fe-N of $\mathbf{1}$ and 2. Also, the shorter bond lengths enhance the interaction between the ligands and iron(II) metal center. In terms of the bond angles, the angles of terpyridine of the different spin excited state structures still gradually decrease, with small deviations ( 0.7 to 1.4 degrees), in the order of quintet, triplet and singlet. The changing rules of the bond lengths and bond angles may result from the Jahn-Teller effect. The repulsion between the $d$ orbitals of iron(II) and the $n / \pi$ orbitals of terpyridine result in the extension of the Fe-N bonds of 1 and 2 .

Compared with $\left[\mathrm{Fe}(\mathrm{tpy})_{2}\right]^{2+}, \mathrm{Fe}-\mathrm{N}_{\mathrm{t}}$ and $\mathrm{N}_{\mathrm{t}}-\mathrm{N}_{\mathrm{c}}-\mathrm{N}_{\mathrm{t}}$ of $\mathbf{1}$ and 2 show obvious extension due to the halogen substituents. $\left[\mathrm{Fe}(\mathrm{tpy})_{2}\right]^{2+}$ has only $0.01 \AA$ deviations of $\mathrm{Fe}-\mathrm{N}_{\mathrm{c}}$ in the singlet and triplet states from $\left[\mathrm{Fe}(\mathrm{dxtpy})_{2}\right]^{2+}$. However, the bond lengths of Fe- $\mathrm{N}_{\mathrm{t}}$ of $\left[\mathrm{Fe}(\mathrm{tpy})_{2}\right]^{2+}$ are 0.09 to $0.14 \AA$ longer than those of $\left[\mathrm{Fe}(\mathrm{dxtpy})_{2}\right]^{2+}$ due to the substitution of $\mathrm{Br}$ and $\mathrm{Cl}$. The singlet ground state of $\left[\mathrm{Fe}(\mathrm{tpy})_{2}\right]^{2+}$ may result from the increased ligand field split, which improves the energy gap of the $e_{g}$ and $t_{2 g}$ orbitals. In comparison with $\left[\mathrm{Fe}(\mathrm{tpy})_{2}\right]^{2+}$, the octahedral structures of $\mathbf{1}$ and $\mathbf{2}$ are more twisted, which weakens the interaction between iron(II) and terpyridine because there is less orbital overlap among the $d$ orbitals and $n / \pi$ orbitals. In addition, the dihedral angles of the single terpyridine and two terpyridines separately increase from quintet to triplet to singlet, which may decrease the effective radiative process ands increase the nonradiative decay. Furthermore, for the dihedral angles of the single terpyridine, the large deviations of 12.4 to 15.8 degrees between 1 and 2 in different spin excited states indicate that the replacement by bromine can result in larger distortion of the structures and change the population of the potential energy surfaces. Furthermore, $\theta$, the bond angle between the iron(II) center and central nitrogen atoms, shows that the larger distortion of the structures of $\mathbf{1}$ than of $\mathbf{2}$ may be caused by the replacement of bromine. Concerning the bond lengths of $\mathrm{Fe}-\mathrm{X}$ and $\mathrm{X}$-pyridyl, the larger bromine atom has greater steric repulsion interactions and weaker influence on the iron(II) center. We found that the geometries of the quintet, triplet and singlet states have the same average values of their bond lengths and bond angles. However, the geometrical characteristics of the septet states of the two complexes are obviously special because one $\beta$ electron is flipped onto the $\pi^{*}$ of the closer ligand as $\alpha$ spin. $400 \mathrm{~nm}$ excitation can easily cause the complexes to reach the energy levels of the septet states. The deactivating pathway beginning from ${ }^{7}$ MLCT will be discussed in the next part.

\subsection{Absorption properties and hole-electron analysis}

$\left[\mathrm{Fe}(\mathrm{tpy})_{2}\right]^{2+}$ with the singlet ground state has been theoretically proved by the Jakubikova et $a .^{\mathbf{4 4}}$ The differences in the absorption properties between the singlet ground state and quintet ground states are another interesting topic that may be

Table 1 The optimized geometry data of the different spin excited states of $\left[\mathrm{Fe}(\mathrm{tpy})_{2}\right]^{2+}, 1$ and 2 . The rows of Exp. show the data of the X-ray crystal structures

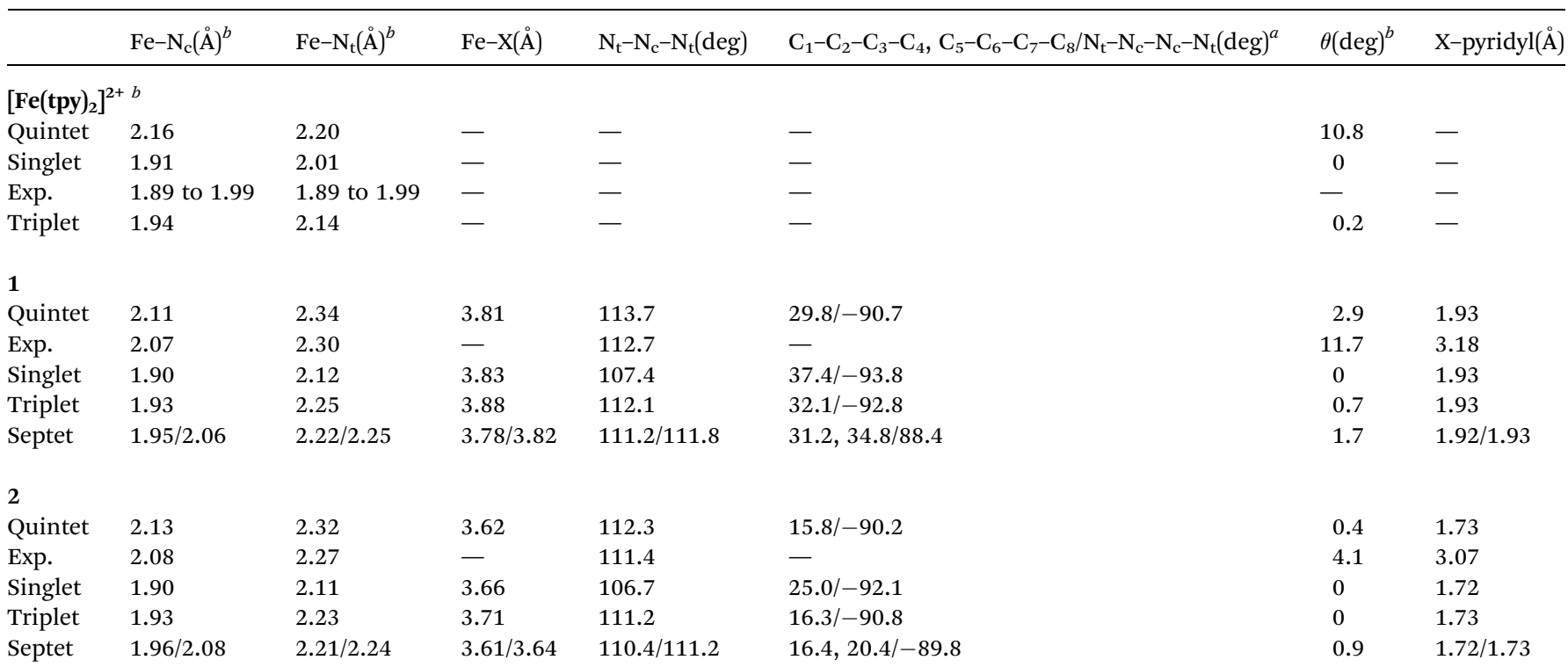

${ }^{a} \mathrm{~N}_{\mathrm{c}}$ is the central nitrogen atom of tpy. $\mathrm{N}_{\mathrm{t}}$ represents the terminal nitrogen atoms of tpy. $\mathrm{X}$ is the halogen atoms. The line of dihedrals includes the average dihedrals of single dxtpy (positive values) and the dihedrals between two dxtpy ligands. $\theta$ is the bond angle between the iron(II) metal center and the central nitrogen atoms of the ligands. All the bond lengths are average values, except for Fe- $\mathrm{N}_{\mathrm{c}}$ of the septet states. ${ }^{b}$ See ref. 12 and 42 . 
investigated in the future. Herein, only the absorption properties based on the optimized quintet ground states of $\mathbf{1}$ and $\mathbf{2}$ are discussed. The electron transition configurations, excitation energies, oscillator strengths $(f)$, and assignments of the main absorption bands are listed in Table S1. $\uparrow$ The corresponding absorption spectra of $\mathbf{1}$ and $\mathbf{2}$ and the alpha and beta spin molecular orbitals are displayed in Fig. 2 and 3.

The calculated absorption spectra are in good agreement with the experimental values. ${ }^{6}$ It is obvious that the bromine substituents result in a slight blue-shift of the lowest peak compared to the chlorine substituents. The lowest absorption peak of $\mathbf{1}$ is mainly contributed by $\mathrm{Q}_{10}$, with the transitions of $\mathrm{H}(\mathrm{B}) \rightarrow \mathrm{L}(\mathrm{B})$ and $\mathrm{H}(\mathrm{B}) \rightarrow \mathrm{L}+5(\mathrm{~B})$. The assignment of $\mathrm{H}(\mathrm{B}) \rightarrow$ $\mathrm{L}(\mathrm{B})$ is MLCT/LLCT (ligand-to-ligand charge transfer) and that of $\mathrm{H}(\mathrm{B}) \rightarrow \mathrm{L}+5(\mathrm{~B})$ is MLCT/LLCT/MC. However, the lowest absorption peak of 2 is mainly contributed by $\mathrm{Q}_{7}$ with the transitions of $\mathrm{H}(\mathrm{B}) \rightarrow \mathrm{L}(\mathrm{B})$, whose assignment is MLCT/MC. The hypsochromic shift may result from the increase of LLCT. Meanwhile, the strongest absorption peaks of 1 and 2 consist of similar high excited states to almost degenerate energy levels. Therefore, they have similar strongest absorption peaks to the main transition configuration of ILCT (intraligand charge transfer).

Nevertheless, there are too many transition configurations with trivial proportions because of the special quintet ground state excitations, although we have a rough conclusion about the absorption properties. The hole and electron analysis performed with Multiwfn can conclude and transfer the manifold orbital transition characters into one main transition contribution, such as a natural transition orbital (NTO). The corresponding data and visual pictures are displayed in Table 2 and Fig. 4, where $D$ is the total magnitude of the charge transfer length between the centroids of the hole and electron and $t$ is the overall measure of the separation degree of the hole and electron in the charge transfer (CT) direction, which is from electrons to holes. The negative values of $t$ and small values of $D$ show that the separation degrees of the hole and electron in the CT direction of $\mathbf{1}$ and $\mathbf{2}$ are very small. The $D$ of the quintet excited state $\mathrm{Q}_{10}$ of 1 is $0.04 \AA$ longer than that of the quintet excited state $\mathrm{Q}_{7}$ of 2 . The absolute value of $t$ of $\mathrm{Q}_{10}$ of $\mathbf{1}$ is about $0.11 \AA$ longer than that of $\mathrm{Q}_{7}$ of 2 . This indicates that the $\mathrm{CT}$

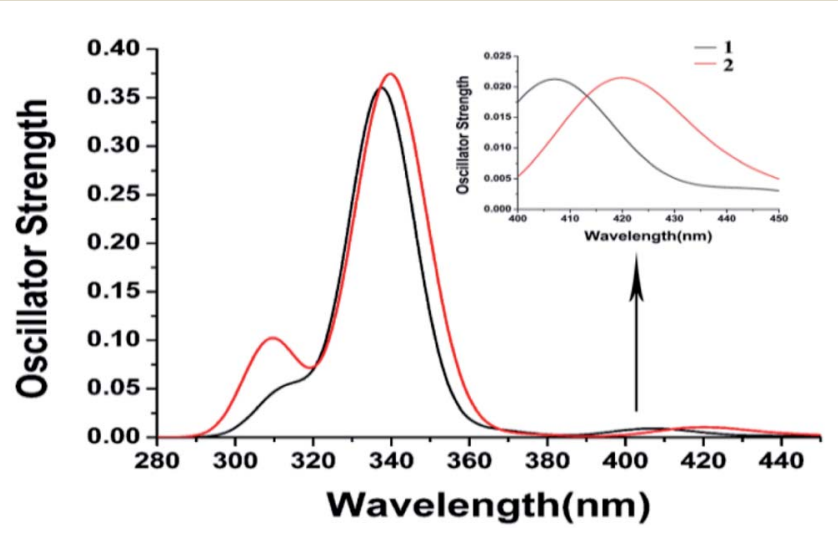

Fig. 2 Calculated absorption spectra and enlargement of the lowest absorption peaks of 1 and 2 .
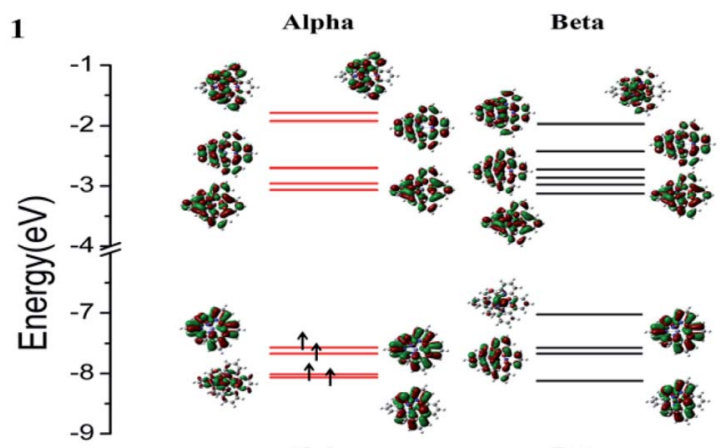

$\mathbf{2}$
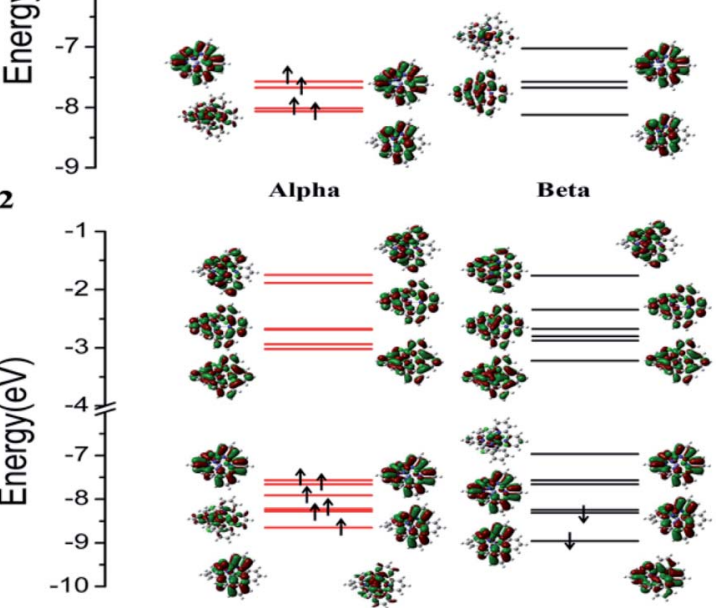

Fig. 3 The main alpha and beta spin molecular orbitals of 1 and 2

process of $\mathbf{1}$ is more obvious, which may result in the hypsochromic shift of the lowest absorption peak of 1. $S_{\mathrm{r}}$ is the geometric average of the overlap function between the hole and electron distribution. The range of $S_{\mathrm{r}}$ values is $[0,1]$. This indicates complete overlap of the hole and electron when $S_{\mathrm{r}}$ is 1 . So, the overlap extent of $\mathrm{Q}_{10}$ of $\mathbf{1}$ is slightly smaller than that of $\mathrm{Q}_{7}$ of 2. $\Delta \sigma$ is the overall root-mean-square deviation of the extent of spatial distribution of the hole and electron. The smaller $\Delta \sigma$ and smaller $S_{\mathrm{r}}$ of $\mathbf{1}$ illustrate that the MLCT from the

Table 2 Detailed data of the hole and electron analysis of the absorption bands of 1 and $2^{a}$

\begin{tabular}{llllll}
\hline Excited state & $S_{\mathrm{r}}$ & $D(\AA)$ & $t(\AA)$ & $\Delta \sigma(\AA)$ & Assignment \\
\hline
\end{tabular}

1

$\mathrm{Q}_{0} \rightarrow \mathrm{Q}_{6} \quad 0.647 \quad 0.006 \quad-2.079 \quad 0.292 \quad$ MLCT/LLCT $(82.3 \%)$ $\mathrm{Q}_{0} \rightarrow \mathrm{Q}_{10} \quad 0.453 \quad 0.048 \quad-1.674 \quad 1.697 \quad$ MLCT/LLCT $(88.7 \%)$ $\mathrm{Q}_{0} \rightarrow \mathrm{Q}_{13} \quad 0.533 \quad 0.058 \quad-2.013 \quad-0.566 \quad$ LLCT/MLCT $(84.7 \%)$ $\begin{array}{llllll}\mathrm{Q}_{0} \rightarrow \mathrm{Q}_{14} & 0.647 & 0.092 & -2.062 & -0.072 & \text { LLCT }(93.7 \%)\end{array}$

2

$\mathrm{Q}_{0} \rightarrow \mathrm{Q}_{5} \quad 0.672 \quad 0.009 \quad-2.077 \quad 0.376 \quad$ ILCT $(85.4 \%)$

$\mathrm{Q}_{0} \rightarrow \mathrm{Q}_{7} \quad 0.497 \quad 0.008 \quad-1.568 \quad 1.754 \quad$ MLCT/LLCT $(92.9 \%)$

$\mathrm{Q}_{0} \rightarrow \mathrm{Q}_{13} \quad 0.611 \quad 0.013 \quad-2.198 \quad-0.241 \quad$ MLCT/LLCT $(86.4 \%)$

$\mathrm{Q}_{0} \rightarrow \mathrm{Q}_{14} \quad 0.515 \quad 0.021 \quad-1.902 \quad 1.340 \quad$ MLCT/LLCT $(94.4 \%)$

$\mathrm{Q}_{0} \rightarrow \mathrm{Q}_{15} \quad 0.621 \quad 0.021 \quad-2.155 \quad-0.358 \quad$ MLCT/LLCT $(88.2 \%)$

a $S_{\mathrm{r}}$ is the geometric average of the overlap function between the hole and electron distributions. $D$ is the total magnitude of the charge transfer length between the centroids of the hole and electron. $t$ is the overall measure of the separation degree of the hole and electron in the charge transfer direction. $\Delta \sigma$ is the overall root-mean-square deviation of the extent of spatial distribution of the hole and electron. ILCT/LLCT represent intraligand/ligand-to-ligand charge transfer. LMCT/MLCT are ligand-to-metal/metal-to-ligand charge transfer. MC represents metal-center charge transfer. 


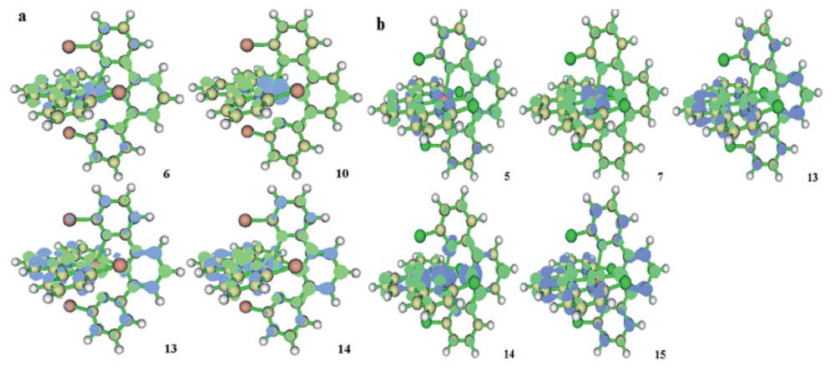

Fig. 4 The hole and electron populations of 1 (a) and 2 (b). The numbers at the bottom of each structure show the different quintet excited states. Blue indicates the population of holes. Green represents the population of electrons. The charge direction is from hole to electron.

$\mathrm{d}_{y z}$ of iron(II) ion to the $\pi^{*}$ of tpy of $\mathbf{1}$ is more distinct. Therefore, the hypochromatic shift from 2 to 1 depends on a stronger CT process. The charge transfer process based on a single molecule occurs before exciton recombination among polymolecules. We will simulate exciton recombination in a future article. In this work, the charge transfer state and excited state relaxation pathways are discussed as the main points.

\subsection{The potential energy curves (PECs) of 1 and 2}

Although the reason for the differences in the lowest absorption peaks has been explained, the deviation of the excited state lifetimes of $\mathbf{1}$ and $\mathbf{2}$ is still obscure. Thus, the PECs with the same values of $\mathrm{Fe}-\mathrm{N}_{\mathrm{c}}$ in Fig. 5 were used to qualitatively explore the corresponding decay mechanism. The detailed data of the PECs are listed in Table S2. $\dagger$ It is obvious that the energy gaps among the PECs of ${ }^{1} \mathrm{MLCT},{ }^{3} \mathrm{MC}$ and ${ }^{5} \mathrm{MC}$ of $\mathbf{1}$ are slightly larger than those of 2. Meanwhile, the 1.4 ps lifetime deviation between $\mathbf{1}$ and 2 may result from the intersystem crossing among the PECs of the different spin states. If we follow Kasha's rule and the FrankCondon rule, the order of intersystem crossing will be from septet to singlet to triplet to quintet, and all the intersystem crossing will occur from the minimum points of the previous PECs to the points with the same structures of the next PECs. If the decay path is ${ }^{7}$ MLCT $-{ }^{5}$ MLCT $-{ }^{3}$ MLCT $-{ }^{1}$ MLCT through crossing of the potential energy surfaces, it may be difficult due to probable high minimum energy crossing points and large structural distortion in the ultrafast process. In contrast, the transition by vertical decay in the Franck-Condon regions may be feasible because of the more distinct $\mathrm{d}$ orbital degeneration caused by the tridentate
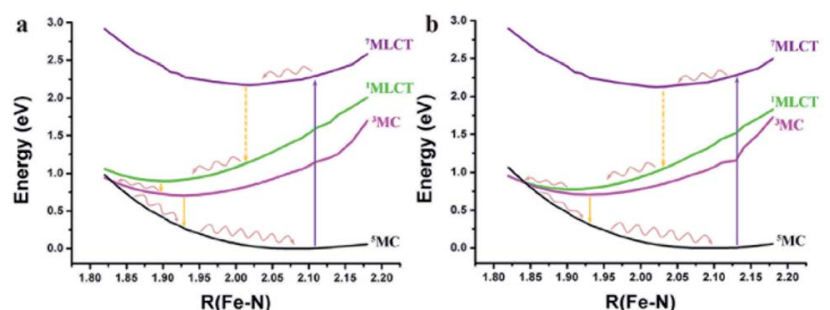

Fig. 5 The PECs of (a) 1 and (b) 2 consisting of the average values of two $\mathrm{Fe}-\mathrm{N}_{\mathrm{c}}$. ligand tpy than by the bidentate ligand. ${ }^{45}$ Therefore, we think a vertical transition from the high spin excited states in the Franck-Condon regions may be easier than the deactivated process. The smaller energy gap of 2 results in a faster vertical decay process than in 1 (in Table S2 $\dagger$ ). Furthermore, the energy crossing point between the singlet and quintet states of 1 is much higher than that of 2 . The excitons decay from ${ }^{7}$ MLCT by a vertical process and rapidly relax to the minimum points. However, the energy crossing points among ${ }^{1} \mathrm{MLCT},{ }^{3} \mathrm{MC}$ and ${ }^{5} \mathrm{MC}$ of 2 are very close, and the deactivation process may directly go through the crossing point between ${ }^{1} \mathrm{MLCT}$ and ${ }^{5} \mathrm{MC}$ as non-radiative decay. Compared with 2, a higher energy crossing point between ${ }^{1}$ MLCT and ${ }^{5} \mathrm{MC}$ of 1 can effectively decrease this process and promote the radiative decay, which will increase the excited state lifetime.

\subsection{The analysis of the transition state coupled with natural orbitals for chemical valence (ETS-NOCV)}

To explore the interactions between the metal centers and the terpyridines quantitatively, ETS-NOCV analysis was performed. All the detailed ETS-NOCV data of the singlet states of $\mathbf{1}$ and $\mathbf{2}$ are listed in Table 3. The graphical information of the deformation densities of different pairwise orbital interactions of the corresponding modes of $\mathbf{1}$ and $\mathbf{2}$ are displayed in Fig. 6. ETSNOCV is an effective and convenient method to analyze the decomposition of the interaction energy $\left(\Delta E_{\text {int }}\right)$ between the fragments of molecules, where $\Delta E_{\text {int }}$ can be expressed as follows:

$$
\Delta E_{\text {int }}=\Delta E_{\text {pauli }}+\Delta E_{\text {elstat }}+\Delta E_{\text {orb }}
$$

$\Delta E_{\text {pauli }}$ is the exchange (Pauli) repulsion energy. $\Delta E_{\text {elstat }}$ is the quasiclassical Coulomb interaction. $\Delta E_{\text {orb }}$ originates from the orbital mixing and is identified as the covalent contribution to the chemical bond. In energy and visualization, $\Delta E_{\text {orb }}$ developed by Ziegler and Rauk can be analyzed to determine which part of the orbital energy is involved in the metal-ligand interactions. The deformation density $\Delta \rho$ in the NOCV representation is a sum of pairs of complimentary eigenfunctions $\left(\psi_{-k}, \psi_{k}\right)$ corresponding to the eigenvalues $-\lambda_{k}$ and $\lambda_{k}$ as follows:

Table 3 Energy decomposition analysis and energetic estimate of each deformation density $\Delta \rho . \Delta E_{\text {pauli }}$ is the exchange (Pauli) repulsion energy. $\Delta E_{\text {elstat }}$ is the quasiclassical coulomb interaction. $\Delta E_{\text {orb }}$ arises from the orbital mixing and is identified as the covalent contribution to the chemical bond

\begin{tabular}{lll}
\hline & $\mathbf{1}$ & $\mathbf{2}$ \\
\hline$\Delta E_{\text {int }}$ & -499.2 & -507.9 \\
$\Delta E_{\text {pauli }}$ & 330.6 & 333.7 \\
$\Delta E_{\text {elstat }}$ & $-345.1(41.6 \%)$ & $-355.8(42.3 \%)$ \\
$\Delta E_{\text {orb }}$ & $-484.7(58.4 \%)$ & $-485.8(57.7 \%)$ \\
$\Delta E_{1}$ & $-107.4(22.2 \%)$ & $-110.8(22.8 \%)$ \\
$\Delta E_{2}$ & $-115.7(23.9 \%)$ & $-114.3(23.5 \%)$ \\
$\Delta E_{9}$ & $-19.4(4.0 \%)$ & $-19.8(4.1 \%)$ \\
$\Delta E_{\text {rest }}$ & $-261.6(49.9 \%)$ & $-260.7(49.6 \%)$
\end{tabular}




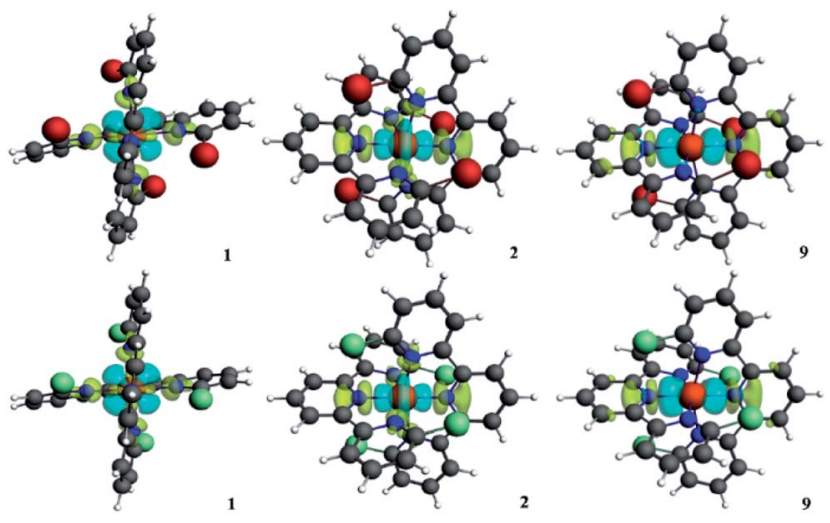

Fig. 6 Plots of the deformation density $\Delta \rho$ of the pairwise orbital interactions. 1 is at the top and 2 is at the bottom. The numbers at the bottom of each construction represent the different modes of deformation density. The direction of the charge flow is from yellow to cyan.

$$
\Delta \rho=\Sigma \Delta \rho_{k}(\mathrm{r})=\Sigma \lambda_{k}\left(-\psi_{-k}^{2}(\mathrm{r})+\psi_{k}^{2}\right)
$$

where $k$ goes over the pair of NOCV. The pictures of the deformation density plots are accompanied with the energetic estimations $\left(\Delta E_{\text {orb }}(k)\right)$ for each $\Delta \rho_{k}$ :

$$
\Delta E_{\mathrm{orb}}=\Sigma \Delta E_{k}^{\mathrm{orb}}=\Sigma \lambda_{k}\left(-F_{-k}^{\mathrm{TS}}+F_{k}^{\mathrm{TS}}\right)
$$

where $-F_{-k}^{\mathrm{TS}}$ and $F_{k}^{\mathrm{TS}}$ are the diagonal transition-state KohnSham matrix elements corresponding to the NOCV with eigenvalues of $-\lambda_{k}$ and $\lambda_{k}$, respectively.

In terms of $\Delta E_{\text {int }}$, we found that the metal-ligand interactions of $\mathbf{1}$ are $8.7 \mathrm{kcal} \mathrm{mol}^{-1}$ weaker than those of 2 . The occupied $3 \mathrm{~d}$ orbitals of the iron(II) center encounter not only the stabilizing interaction of the $\mathrm{n}$ orbital on the 6 nitrogen atoms of tpy, but also the repulsive interaction with the occupied $2 \mathrm{~s}$ and $2 p$ orbitals on the other centers. As a result, the additional dctpys of 2 result in stronger electrostatic attraction and weaker Pauli repulsion. However, this does not play a significant role in enhancing the orbital interactions. The deviation of $\Delta E_{\text {orb }}$ between 1 and 2 is only $1.1 \mathrm{kcal} \mathrm{mol}^{-1}$. Due to the stronger nonmetallic character of $\mathrm{Cl}$ and the longer bond lengths of $\mathrm{Fe}-\mathrm{N}$ in 2, the interactions between Fe and the ligand may lie in a strong electrostatic attraction state and weak repulsion state on the interaction potential curves.

Also, there are similar pairwise orbital interactions. In Fig. 6, we can find that the main orbital contributions of the singlet states of 1 and 2 originate from $\mathrm{d}_{x^{2}-y^{2}}$ of iron(II) ion and the $n$ orbitals of the nitrogen atoms to $\mathrm{d}_{z^{2}}$ or $\mathrm{d}_{x y}$ of iron(II) ion as $\sigma^{*}$ or non-bonding with similar contributions. Thus, we found that the substitution of bromine and chlorine impacts the metalligand interactions by electrostatic attraction to a greater extent.

\section{Conclusions}

1 and 2 were studied to explore how different halogen atoms impact the MLCT lifetimes by a combination of electronic structures, absorption spectra, ETS-NOCV and PECs. The larger distortion of 1 than 2 is caused by the sterically hindered interactions of bromine atoms. Taking the advantage of the combination of the Kohn-Sham molecular orbitals of the absorption properties and the hole and electron analysis, the increase in the CT proportion from the chlorine to bromine substituents causes a hypsochromic shift of the lowest absorption peaks. The PECs show that the total energy gaps of intersystem crossing following the Franck-Condon rule and Kasha's rule on the PECs of the different spin states of $\mathbf{1}$ are larger than those of 2 , which results in the 1.4 ps longer lifetime. The higher energy crossing points of 1 improve the energy barriers and hinder the thermodynamic hopping from the optimized structures to the energy crossing points, which results in faster non-radiative decay. ETS-NOCV and the pictures of the deformation density show that the substitution of bromine atoms will decrease the electrostatic attractions between the metal and ligands but will slightly impact the orbital interactions. Unfortunately, the analysis of the MLCT progress and lifetimes is only qualitative. As different metal center analogues, $\left[\mathrm{Ru}(\mathrm{tpy})_{2}\right]^{2+}$ with $602 \mathrm{~nm}$ emission at $77 \mathrm{~K}$ only has a singlet ground state, a triplet metal-ligand charge transfer state $\left({ }^{3} \mathrm{MLCT}\right)$ and a triplet metal center state $\left({ }^{3} \mathrm{MC}\right) \cdot{ }^{46}$ However, $\left[\mathrm{Fe}(\mathrm{dxtpy})_{2}\right]^{2+}$ without luminescence in this article has ground quintet states, singlet metal-ligand charge transfer states $\left({ }^{1} \mathrm{MLCT}\right)$, and ${ }^{3} \mathrm{MC}$ and septet metal-ligand charge transfer states ( ${ }^{7}$ MLCT). Due to the abundant excited states, exploration of the complicated excited states of the deactivated pathways in detail is difficult. The photophysical properties of the complex containing fluorine still remain to be discussed. We will make more effort to resolve these problems and explore more properties and mechanisms of iron complexes in the future.

\section{Conflicts of interest}

There are no conflicts to declare.

\section{Acknowledgements}

This work is funded by the Natural Science Foundation of China (Grant Nos. 21873038 and 21573088), and the "13th Five-Year" science and technology project of the education department of Jilin province (Grant No. JJKH20180123KJ) and the Young Scholar Training Program of Jilin University.

\section{References}

1 J.-Y. Hung, C.-H. Lin, Y. Chi, M.-W. Chung, Y.-J. Chen, G.-H. Lee, P.-T. Chou, C.-C. Chen and C.-C. Wu, J. Mater. Chem., 2010, 20, 7682.

2 G. Cheng, S. C. F. Kui, W.-H. Ang, M.-Y. Ko, P.-K. Chow, C.-L. Kwong, C.-C. Kwok, C. Ma, X. Guan, K.-H. Low, S.-J. Su and C.-M. Che, Chem. Sci., 2014, 5, 4819-4830.

3 K. M. Maness, R. H. Terrill, T. J. Meyer, R. W. Murray and R. M. Wightman, J. Am. Chem. Soc., 1996, 118, 10609-10616.

4 Y. Wang, J. Wang, H.-X. Zhang, I. M. Szilágyi and F.-Q. Bai, Organometallics, 2018, 37, 2491-2499.

5 Z.-X. Zhang, W. Wei, F.-Q. Bai, S. Bibi and H.-X. Zhang, Org. Chem. Front., 2017, 4, 2191-2201. 
6 S. M. Fatur, S. G. Shepard, R. F. Higgins, M. P. Shores and N. H. Damrauer, J. Am. Chem. Soc., 2017, 139, 4493-4505.

7 M. Abrahamsson, H. C. Becker and L. Hammarstrom, Dalton Trans., 2017, 46, 13314-13321.

8 T. He, A. Ren, S. Chen, Z. Yang, P. Yu, Y. Chen, X. Ding, L. Shen, H. Zhang and L. Zou, Org. Electron., 2017, 45, 9-19.

9 W. Zhang, K. S. Kjaer, R. Alonso-Mori, U. Bergmann, M. Chollet, L. A. Fredin, R. G. Hadt, R. W. Hartsock, T. Harlang, T. Kroll, K. Kubicek, H. T. Lemke, H. W. Liang, Y. Liu, M. M. Nielsen, P. Persson, J. S. Robinson, E. I. Solomon, Z. Sun, D. Sokaras, T. B. van Driel, T. C. Weng, D. Zhu, K. Warnmark, V. Sundstrom and K. J. Gaffney, Chem. Sci., 2017, 8, 515-523.

10 Y. Liu, T. Harlang, S. E. Canton, P. Chabera, K. SuarezAlcantara, A. Fleckhaus, D. A. Vithanage, E. Goransson, A. Corani, R. Lomoth, V. Sundstrom and K. Warnmark, Chem. Commun., 2013, 49, 6412-6414.

11 X. C. Ma, Y. Dai, L. Yu and B. B. Huang, Light Sci Appl, 2016, 5, e16017.

12 X. Wang, F.-Q. Bai, Y.-T. Liu, J. Wang and H.-X. Zhang, Synth. Met., 2016, 213, 18-24.

13 A. Baker and H. Goodwin, Aust. J. Chem., 1985, 38, 207-214. 14 L. A. Buldt and O. S. Wenger, Dalton Trans., 2017, 46, 1517515177.

15 I. M. Dixon, F. Alary, M. Boggio-Pasqua and J. L. Heully, Dalton Trans., 2015, 44, 13498-13503.

16 W. Zhang, Y. Li, V. P. Nguyen, Z. Huang, Z. Liu, X. Wang and Y. M. Paulus, Light Sci Appl, 2018, 7, 103.

17 G. Auböck and M. Chergui, Nat. Chem., 2015, 7, 629.

18 A. Cannizzo, C. J. Milne, C. Consani, W. Gawelda, C. Bressler, F. van Mourik and M. Chergui, Coordin. Chem. Rev., 2010, 254, 2677-2686.

19 W. Zhang, R. Alonso-Mori, U. Bergmann, C. Bressler, M. Chollet, A. Galler, W. Gawelda, R. G. Hadt, R. W. Hartsock, T. Kroll, K. S. Kjær, K. Kubiček, H. T. Lemke, H. W. Liang, D. A. Meyer, M. M. Nielsen, C. Purser, J. S. Robinson, E. I. Solomon, Z. Sun, D. Sokaras, T. B. van Driel, G. Vankó, T.-C. Weng, D. Zhu and K. J. Gaffney, Nature, 2014, 509, 345.

20 L. Liu, T. Duchanois, T. Etienne, A. Monari, M. Beley, X. Assfeld, S. Haacke and P. C. Gros, Phys. Chem. Chem. Phys., 2016, 18, 12550-12556.

21 C. Adamo and V. Barone, J. Chem. Phys., 1999, 110, 61586170.

22 P. J. Hay and W. R. Wadt, J. Chem. Phys., 1985, 82, 299-310.

23 G. A. Petersson, A. Bennett, T. G. Tensfeldt, M. A. Al-Laham, W. A. Shirley and J. Mantzaris, J. Chem. Phys., 1988, 89, 21932218.

24 L. E. Roy, P. J. Hay and R. L. Martin, J. Chem. Theory Comput., 2008, 4, 1029-1031.

25 G. Scalmani and M. J. Frisch, J. Chem. Phys., 2010, 132, 114110.

26 A. W. Ehlers, M. Böhme, S. Dapprich, A. Gobbi, A. Höllwarth, V. Jonas, K. F. Köhler, R. Stegmann, A. Veldkamp and G. Frenking, Chem. Phys. Lett., 1993, 208, 111-114.
27 R. Krishnan, J. S. Binkley, R. Seeger and J. A. Pople, J. Chem. Phys., 1980, 72, 650-654.

28 M. J. Frisch, G. W. Trucks, H. B. Schlegel, G. E. Scuseria, M. A. Robb, J. R. Cheeseman, G. Scalmani, V. Barone, G. A. Petersson, H. Nakatsuji, X. Li, M. Caricato, A. V. Marenich, J. Bloino, B. G. Janesko, R. Gomperts, B. Mennucci, H. P. Hratchian, J. V. Ortiz, A. F. Izmaylov, J. L. Sonnenberg, Williams, F. Ding, F. Lipparini, F. Egidi, J. Goings, B. Peng, A. Petrone, T. Henderson, D. Ranasinghe, V. G. Zakrzewski, J. Gao, N. Rega, G. Zheng, W. Liang, M. Hada, M. Ehara, K. Toyota, R. Fukuda, J. Hasegawa, M. Ishida, T. Nakajima, Y. Honda, O. Kitao, H. Nakai, T. Vreven, K. Throssell, J. A. Montgomery, J. E. Peralta, F. Ogliaro, M. J. Bearpark, J. J. Heyd, E. N. Brothers, K. N. Kudin, V. N. Staroverov, T. A. Keith, R. Kobayashi, J. Normand, K. Raghavachari, A. P. Rendell, J. C. Burant, S. S. Iyengar, J. Tomasi, M. Cossi, J. M. Millam, M. Klene, C. Adamo, R. Cammi, J. W. Ochterski, R. L. Martin, K. Morokuma, O. Farkas, J. B. Foresman and D. J. Fox, Gaussian 16 Rev. A.03, Wallingford, CT, 2016.

29 T. Lu and F. Chen, J. Comput. Chem., 2012, 33, 580-592. 30 T. Ziegler and A. Rauk, Theore. Chim. Acta, 1977, 46, 1-10. 31 T. Ziegler and A. Rauk, Inorg. Chem., 1979, 18, 1755-1759. 32 T. Ziegler and A. Rauk, Inorg. Chem., 1979, 18, 1558-1565.

33 M. P. Mitoraj, A. Michalak and T. Ziegler, J. Chem. Theory Comput., 2009, 5, 962-975.

34 C. Fonseca Guerra, J. G. Snijders, G. te Velde and E. J. Baerends, Theore. Chem. Acc., 1998, 99, 391-403.

35 G. te Velde, F. M. Bickelhaupt, E. J. Baerends, C. Fonseca Guerra, S. J. A. van Gisbergen, J. G. Snijders and T. Ziegler, J. Comput. Chem., 2001, 22, 931-967.

36 A. D. Becke, Phys. Rev. A: At., Mol., Opt. Phys., 1988, 38, 30983100.

37 J. P. Perdew, Phys. Rev. B: Condens. Matter Mater. Phys., 1986, 33, 8822-8824.

38 J. P. Perdew, Phys. Rev. B: Condens. Matter Mater. Phys., 1986, 34, 7406.

39 E. Van Lenthe and E. J. Baerends, J. Comput. Chem., 2003, 24, 1142-1156.

40 E. v. Lenthe, E. J. Baerends and J. G. Snijders, J. Chem. Phys., 1993, 99, 4597-4610.

41 E. v. Lenthe, E. J. Baerends and J. G. Snijders, J. Chem. Phys., 1994, 101, 9783-9792.

42 E. v. Lenthe, A. Ehlers and E.-J. Baerends, J. Chem. Phys., 1999, 110, 8943-8953.

43 C. C. Pye and T. Ziegler, Theore. Chem. Acc., 1999, 101, 396408.

44 J. Nance, D. N. Bowman, S. Mukherjee, C. T. Kelley and E. Jakubikova, Inorg. Chem., 2015, 54, 11259-11268.

45 D. G. Brown, N. Sanguantrakun, B. Schulze, U. S. Schubert and C. P. Berlinguette, J. Am. Chem. Soc., 2012, 134, 12354-12357.

46 A. Soupart, I. M. Dixon, F. Alary and J.-L. Heully, Theor. Chem. Acc., 2018, 137. 